\title{
A Case Study of Political Analysis of Chronic Disease Management System at Outpatient Clinic in Korea
}

\author{
Hyunsook Zin Lee \\ Doctoral student, Department of Nursing, Chung-Ang University \\ zinhslee@gmail.com
}

\begin{abstract}
This article examines the major political challenges associated with chronic disease management system at outpatient clinic using The PolicyMaker program. The article briefly presents major issues on the management of chronic disease in Korea and the efforts of changing chronic disease management system at outpatient clinic that were initiated in 2011.The PolicyMaker method of applied political analysis is described and the results of its application are presented including analysis of the policy content of chronic disease management system at outpatient clinic, and assessments of the positions of major players (private sectors, public sectors, non-governmental organizations and patient group).Given situation, opportunities, obstacles were analyses and strategies were developed. The conclusion presents factors of insufficient negotiation with key players.
\end{abstract}

Keywords: political analysis, chronic disease management system, outpatient clinic

\section{Introduction}

With carelessness establishment of the policy on utilization of healthcare institutions, there has been rivalry between outpatient clinic, general hospital and university hospital, which is so called primary, secondary and tertiary institution respectively. Medical institutions are classified by its size and function in Korea [1]. In case of non-emergency case, patients should have visited firstly primary outpatient clinic and obtained special document for consultation at secondary and tertiary hospitals. This is regulated by the MOHW for the effective use of healthcare resources. However, People go to big and upper level of hospitals for better quality of healthcare service without first visiting primary outpatient clinic because of less credibility on outpatient clinics around their residence. Being convenient for transportation, high level of living standard, no strict regulation on visiting general hospitals without first visiting primary clinic and little gap of medical cost of each institution accelerate people's leaning toward tertiary hospitals [2]. With these phenomena, business environment of outpatient clinics is getting worse condition. People with chronic disease who are required special care for prevention of complications, medication with no omission, regular check-up, and proper guidance for lifestyle modification might be less cared at general hospitals. This is caused by different functions of medical institutes designed by the government. Secondary hospitals are designed for caring in-hospital patients and tertiary hospitals are projected for caring complex patients such as with cancer and researching for advanced medical technology. This means that people with early stage of chronic disease could not receive effective care at secondary and tertiary hospitals. Though patients visit outpatient clinic for primary medical care, many of them visit several different outpatient clinics and see doctors irregularly for their check-ups of chronic disease. Then it is hard to receive effective and consistent medical care.

With ineffective management of healthcare institution and non-effective care of patients with chronic disease, number of patients with diabetes or hypertension who developed complications and hospitalized due to severe complications has recently increased $9.8 \%$ in annual and medical cost had increased from 400 billion Korean won in 
2005 to 3,000 billion Korean won in 2009. It is an example of ineffective use of healthcare resources [2].

There are many reports on issuing the benefits of continuity of care. According to the studies, patients who have usual source of care received more preventive service such as cancer screening $[3,4,5]$. More people had blood pressure tested when having a usual source of care $[4,5,6]$. Several studies reported that people having usual sources of care spend less money and less hospital visits for medical care $[4,5]$.

Given situation, new effective and sustainable healthcare delivery system was necessary. On the occasion, the government announced several policies for better healthcare management system such as reorganization of healthcare institutions by its function and management of chronic disease. Chronic Disease Management System at Outpatient Clinic (hereafter CDMSOC) for the patients with diabetes or hypertension is one of the policies that the government targeted for. It is to clarify the function of each medical institution, to guide people effective use of medical institutes, to advance the management of medical resources, and to increase the quality of medical service. Though it was initiated a few years ago by the government and announced September 2010, when it was implemented on April 2012, it has totally different measures from originally designed due to strong objections and resistance of key players.

\section{Chronic Disease Management System at Outpatient Clinic (CDMSOC)}

Main idea of the policy of CDMSOC is that patient with diabetes or hypertension appoints an outpatient clinic around their residence and visit the doctor after that time for regular medical check-up. Patient who visits the appointed outpatient clinic is expected to receive more qualified medical service such as consistent medical care including monitoring patients' medication, medical tests and education related to disease management and lifestyle modification. At the same time, patient obtains $30 \%$ discount of consultation fee as monetary incentive. The purpose of the policy of CDMSOC is to increase the effectiveness of medical resources utilization and people's quality of life. Through systemic and consistent management of chronic disease by appointed doctor, it expects to reduce the rate of complications, and hospitalization due to uncontrolled condition, and medical cost for managing complications. It starts with patients' asking his or her doctor for application at the first visit of outpatient clinic and the doctor apply it to the National Health Insurance Service (hereafter NHIS) for the patient. The patient then gets monetary benefits, customized education and designed medical care [7].

To doctors who are appointed by patient, they will get monetary benefits of 950 Korean won (almost equivalent to 0.8 US \$) by a patient per visit and special incentives according to the quality of management at the end of certain period from NHIS. Doctors get 2 types of incentive, one is by number of patient that they see and the other is by the quality of patients' management [8]. The former limits maximum 10 times per patient in a year. In order to obtain incentives, the appointed doctor keeps the medical record for each patient and reports it to NHIS regularly. It contains information related to patients' progress. 


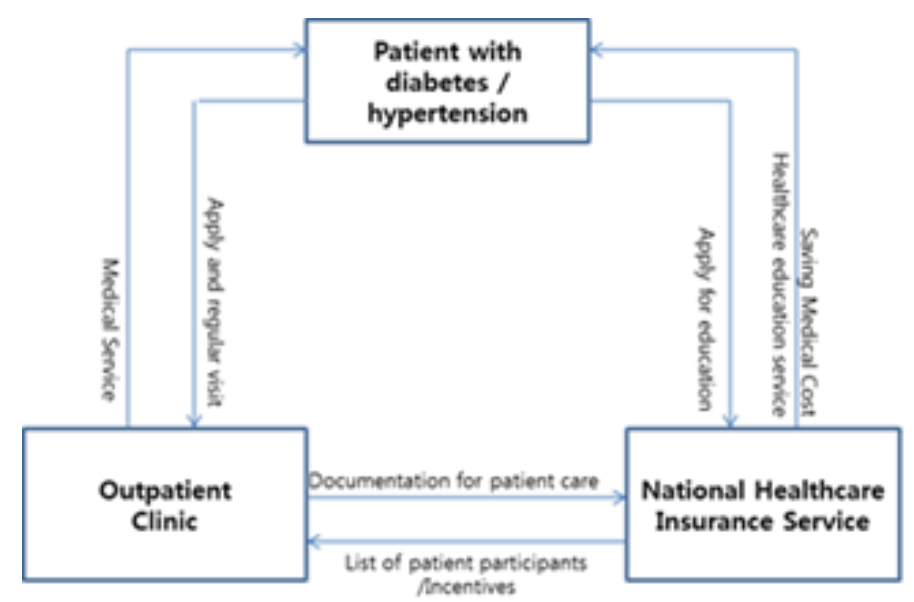

\section{Figure1. Work flow for Chronic Disease Management System at Outpatient Clinic}

The ministry of health and welfare (hereafter MOHW) planned "a clinic per patient" system and doctors' reporting patients' medical record to NHIS. Prior to implementation, doctors were required to receive training for managing patients with diabetes and hypertension. It is mandatory. It is projected to reduce complications and patients' hospitalization due to advanced condition with preventive management of chronic disease

However, the policy implemented on April 2012 was far distant from originally designed by the government. Number of clinics that patient could appoint was changed from one to two, which means "two clinics per patient" and the document recorded and reported by doctor was much simpler with no much information. Doctors participating in this system may not require mandatory training. Most of all, the contents was overlapped the policy existing chronic disease management system.

It has been almost two and half years since its implementation. However there was no attempt to analysis of its politics and impact and the effects of CDMSOC. The purpose of this article is to analysis the politics of CDMSOC and to provide decision-makers relevant information for establishment of healthcare policy in the future.

\section{Method}

The method of applied political analysis known as PolicyMaker (http://www.polimap) was used in this project to assist decision makers in analyzing and managing the policies of CDMSOC. The method provides a systemic analysis of the probable consequences of the proposed policy and the positions of support and opposition taken by key players [9]. Then it assists decision makers in initiating the process to develop strategies for managing the politics of policy reform [10]. It comprises 5 steps of political analysis: 1) It helps to identify the contents of the policy. 2) it supports to find the interests of participants and its relationships, 3) to verify the opportunities and weaknesses, 4) to support strategy development and 5) to evaluate the potential and realistic impacts of the policy [11].

The method helps policy analysts with the political dimensions of policy change. First, the method provides a systemic evaluation of the political environment and a tool to explain the political dimensions of a policy decision. Second, the method provides practical assistance in the development of political strategies. The software included a tool box of 31 expert-suggested political strategies. Third, if conducted with interviews of key stakes holders, the method supports validate the reform group's perceptions about other stakes holders, and assists the reform team view the policy from the perspectives of other stakeholders. The analysis inspires reform groups to make their strategies explicit, and contemplate the strategies, considering the interactions among policies players, and positions [11]. 
This research was based on systemic review of press release from government, position letters of key players, news reports and research papers about chronic disease management system at outpatient clinic.

\section{Results}

\subsection{Stakeholders}

\subsubsection{Korea Medical Association}

At the beginning of the government announcement of the policy, Korea Medical Association (hereafter KMA) welcomed the policy with good reasons of improving quality of medical service through effective utilization of medical resources and the improvement of chronic disease management [12]. Most of all, KMA accepted it as a solution for resolving the hardship of outpatient clinics. However, on the course of negotiation, the KMA changed their position from support to opponent to the policy because of conflicts between sub ordinates of KMA.

\section{Clinic Medical Associations (CMA)}

This is a sub ordinate of KMA, which are composed of doctors' group by region and cities. CMA strongly opposed to the policy. They argued that CDMSOC was the policy for preparing the Doctor Appointment System which the government considers as fundamental healthcare delivery system in the future. The government insisted that the policy of CDMSOC is different from. The Doctor Appointment System is believed the prior step of the Lump-Sum Contract which the government gives doctor fixed money for certain number of patients for their medical service. KMA and CMA positioned against the Lump Sum Contract. Another reason they presented was that the preparation or submission of patients' record to NHIS is an interference of their autonomic activities [12].

\section{The Korea Doctors}

This is a subordinate of KMA, comprise of clinicians who work at outpatient clinics, mostly responsible for primary care. They were against the policy because most of patients with diabetes or hypertension would go to the outpatient clinics where internal medicine doctors work at, and then other than internal medicine doctors would not see many patients. Family doctors and internal medicine doctors were supposed to be the beneficiary of the policy of CDMSOC. However, they did not agree on the policy though they were on support position [8].

\section{Korean Physicians' Association}

A subordinate of KMA constitute internal medicine physicians who mostly work at outpatient clinic and they basically agreed on the policy but they could not express their opinion to the public due to possible conflict with other associations. They were expected to be a beneficiary for the policy [15].

\subsubsection{Doctors' Union}

\section{The Korean Doctor's Union}

Doctors' union was one of the strong opponents. They argued that submission of patient's medical records to NHIS was to domesticate doctors and they considered it as an 
interference of doctor's right. They also claimed that giving discount of consultation fee to patients would be concluded as ineffective use medical resources due to patients' hospital shopping [12].

\section{The Korea Intern Resident Association}

This group was also against the policy. They insisted that if the policy is applied, patients are allocated to already existing clinics and as they complete their training and start business, there will not be many patients remained for them. They argued that it is the right to live [12].

\subsubsection{Civic Groups}

The Citizens Coalition of Economic Justice and Health Right Network support the policy of CDMSOC with good reasons of effective use of medical resources and activation of primary medical care. Though they had positions of support, they were not active on the supporting activities [13].

\subsubsection{The Patients Organization}

The Korea Alliance of Patients Organization had no opinion and position. Majority of the organization are the patients group with critical conditions such as leukemia, cancer, HIV/AIDS etc. Compare to these critical conditions of patient, chronic diseases were seemed to minor issues to them. The issue of CDMSOC policy was treated negligibly.

\subsection{Position Map}

Political environment for the policy of CDMSOC were analysis using the PolicyMaker and results are as follows. As reviewing the power distribution of key players, it was weighted to opponents. Though the civic groups placed on the position of support together with the government, the power was not as big as opponents. The lack of involvement of civic groups and patients' organization forced the KMA and doctors to become the main advocates for CDMSOC. This meant that in order to have negotiation power of the government, the supporters had to be more powerful through coalition with supports, supporting civic groups to voice out their position, bringing supporters from non-mobilized group, asking the patients' organizations participation, or weakening the power of opponents.

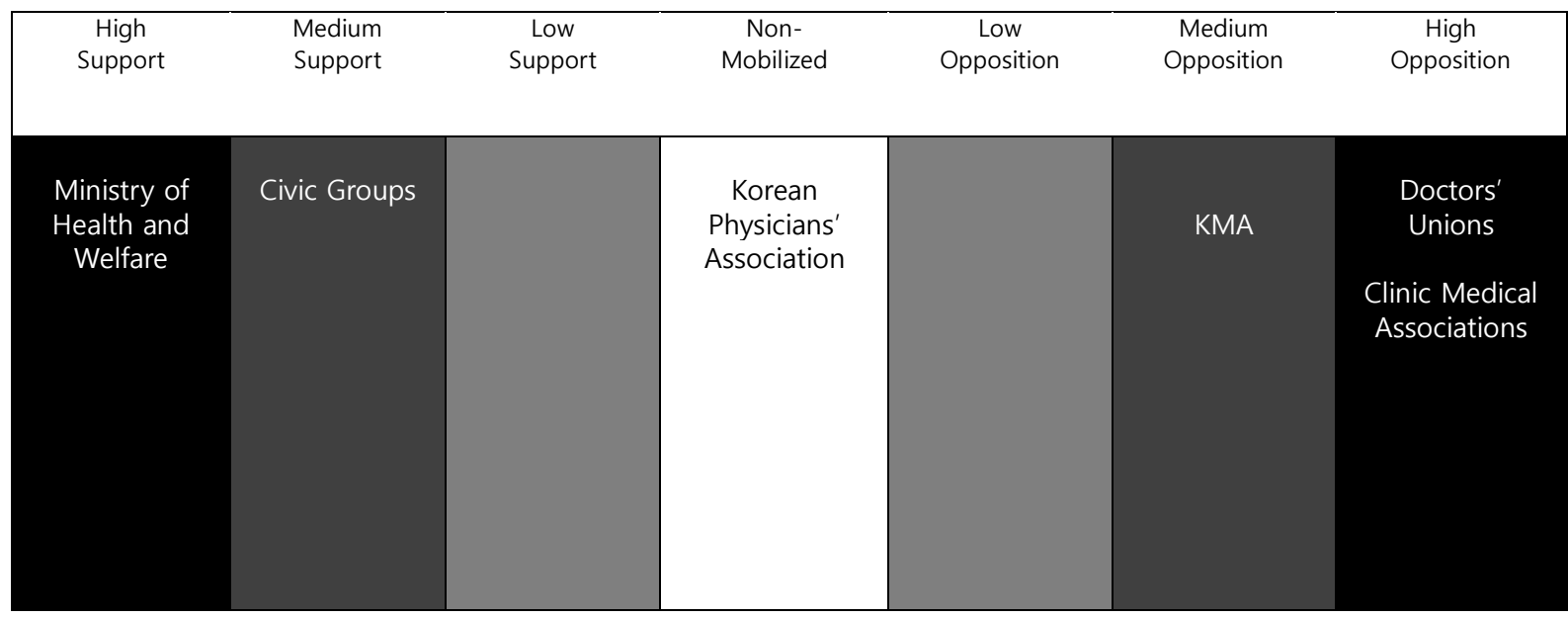

Key to power gradient: white box=low power; gray=medium power; black box= high power

\section{Figure 2. Position Map for Major Players in Chronic Disease Management System at Outpatient Clinic in 2011}




\subsection{Opportunities and Obstacles}

The biggest opportunities were the good reasons of effective use of medial resources with chronic disease management system. There had been issues on the lack of function of healthcare institute, unhealthy healthcare delivery system, ineffective management of chronic disease and medical resources [14]. The civic groups and the government recognized the needs of new policy for the effective management of chronic disease and the activation of primary medical care [13]. Mortality rate of complications of hypertension and diabetes has increased every year and reached $2^{\text {nd }}$ rank in 2009 . Hospitalization due to complications of hypertension and diabetes has increased $9.8 \%$ every year [2]. The government bears the burden of increased medical expense. However, number of people who visit general hospital has increased and over 30 million people visited tertiary hospitals, 1 out 3 were people who can be managed at outpatient clinics. The sense of crisis on being worsened financial status of NHIS made people consider new policy for effective primary care.

However, the obstacles were distrust and strong opposition of medical association which is the suppliers of medical service. Opposition was grounded in mistrust between the government and KMA, which was provoked by the separation of prescribing and dispensing of ethical drugs [9]. The KMA insisted that the policy of CDMSOC was a prior step of preparation of Doctor Appointment System and Lump-Sum Contract System. They said that they could not accept it although the government denied it. The doctor's union argued that the preparation of special documents for patients' medical records, and sending it to NHIS is the invasion of doctor's medical rights and if the government implement the new policy without any amendment, they would refuse medical activities and not to participate to the process of policy.

\subsection{Strategies}

PolicyMaker provides a tool box of 31 basic political strategies for increasing the feasibility of policy reform and a matrix for defining strategy actions and associated risks, problems, and benefits. Strategies are usually designed with the client's full collaboration to assure that the proposed strategies are relevant and realistic under the time and resource constraints. Table 1 provides a summary of the strategy design exercise for CDMSOC in Korea. The MOHW created common ground and vision (Strategy \#1) through the official debate and publication of overall reform of healthcare system. Facing strong objections from KMA and doctor's union, MOHW strengthened the power of supporters, and developed communication channels with KMA to negotiate effectively (Strategy \#2, \#6). The KMA created common goal and mechanism which reduce the intensity of their opposition, which was to establish healthy healthcare delivery system and activate primary health care system (Strategy \#7). At the same time, MOHW needed to identify mediators to negotiate with the Korea Doctors' Association and find acceptable agreement (strategy \#3). With the Korea Physician Association, the government required to persuade this association strengthen their negotiation power and ask KMA agree on the proposed policy. This was possible because the Korea Physician Association would be the most beneficiary of the policy (Strategy \#4). Doctors union was provided the modified contents of the policy which was simpler preparation of document with minimum amount of patients' information, increased number of clinics that patients appoint from 1 to 2, and the removal item of mandatory attendance of doctor's continuing education (Strategies \#5).

Overall, the PolicyMaker exercise produced a set of strategies that could achieved some success for the MOHW. It helped MOHW understand the political environment. 
Table 1. Summary of PolicyMaker Strategies

\begin{tabular}{lll}
\hline Strategy name & \multicolumn{2}{l}{ Actions } \\
\hline Strategy \#1 & 1. & Seek common ground with other organizations \\
$\begin{array}{l}\text { MOHW: } \\
\text { Create common ground }\end{array}$ & 2. & Identity common interests \\
& 3. & Make decisions for opponents easier \\
& 1. & Increase the organizational strength of supports \\
Strategy \#2 & 2. & Develop communicational channel with KMA \\
MOHW: & 3. & Provide experienced staffs \\
Strengthen supports & 4. & Fostering political skills \\
& 1. & Find persuasive mediators to negotiate with opponents \\
Strategy \#3 & 2. & Find acceptable agreement to end their opposition \\
The Korea Doctors Association: & & \\
Persuasive mediation & & \\
Strategy \# 4 & 1. & Persuade supports to strengthen their position \\
Korean Physicians Association: & 2. & Adding more benefits as an incentives \\
Attractive benefits & 3. & Unite with Civic groups to strengthen position \\
Strategy \# 5 & 1. & Persuade opponents to weak their position \\
Doctors Union: & 2. & Adding desired goals or mechanisms to the policy \\
Weaken position & & \\
Strategy \# 6 & 1. & Persuade supports to strengthen their position \\
Civic groups: & 2. & Remind supporters promised benefits compared to \\
Strengthen position & & other policies \\
Strategy \# 7 & & \\
The KMA & 1. & Identify common goals or mechanisms \\
Create common ground & 2. & Reduce the intensity of their opposition \\
\hline & &
\end{tabular}

\section{Conclusions}

This analysis of the political dimensions of CDMSOC in Korea suggests some generalizations that may be relevant to other policy making process. Two factors seemed to affect the pace and feasibility of the CDMSOC proposal in the country in 2012.

CDMSOC was initiated by the MOHW for the purpose of restructuring healthcare system, activation of primary health care system, and effective management of chronic disease. After many twists and turns, it was implemented with the modified measures from the originally designed. The factors affected to this result in were as follows:

\section{The Leadership of the CDMSOC}

The MOHW created the common vision and goals for effective use of healthcare resources and protection people from chronic diseases and it was supported by many people in Korea. However, the measures implemented in April 2012 were modified with several items from the planned and the items amended were seen as important factors for the success of CDMSOC [2]. At the time of the MOHW announced the plan for restructuring healthcare system, the position of Doctors' Union and the Korean Doctors' Association were placed at strong opponent and the power was far weighted to opponents [13]. Two third of physicians were against the policy even though they were interested in. 
The discrepancy between the interest and the attitude was caused by mistrust to the government policy [15]. The doctors' groups which produce medical service, kept a firm position with no change and they threaten the government to stop seeing patients for a while. As the negotiation process was kept going, the MOHW could bring some supports from civic groups and the Korean Physician's Association which could be beneficiary [15]. Though the Korean Physician's Associations could not actively represent their opinion on public, some members agreed on the policy of CDMOC. The Korean Physician's Association could not been officially against the KMA due to a subordinate of the KMA. The Doctors' union and the Korean Physicians Associations soften their opponent position as the MOHW modified some contents of the policy, which were acceptable by the doctors' group [12]. That were change the number of clinic that patients appoint for usual source of care from 1 to 2, the removal of item of mandatory attendance for continuing educational program, and reporting minimum amount of patients' information to NHIS [2]. With this amendment, the CDMSOC became the policy of not much different from already existing policy for diabetes and hypertension management.

\section{Discussion and Limitations}

Distrust of KMA to the government and insufficient organizational power of the government were considered main causes of amending the measures of original form of CDMSOC. Building trust between the parties was considered one of the most important factors to draw successful negotiation and implementation [16]. Since it has been almost 3 years of implement, it is time to evaluate the impact of content amendment and CDMSOC's outcome.

The article presents the political analysis on CDMSOC and the analysis is based on the systemic review of researches, position papers, News articles. It is limitation of this political analysis that there is no interviewing people who were involved in the process of CDMSOC. Therefore, the comments and opinions from key people were not included in this political analysis.

\section{References}

[1] C. R. Shin, "Key Issues of Chronic Disease Management System at outpatient clinic", Medical Policy Forum, vol.10, no.1, (2012), pp.38-43.

[2] "The Ministry of Health and Welfare", Plan for restructuring healthcare institute for sustainable healthcare delivery system focused on health care customer, (2011).

[3] M. Y. Kim, J. H. Kim, I. K. Choi, I. H. Hwang and S. Y. Kim, "Effects of having usual source of care on preventive service and chronic disease control: A systemic review", Korean Journal of Family Medicine, vol.33, no.6, (2012), pp.336-345.

[4] M. D. Cabana and S. H. Jee, "Does continuity of care improve patient outcomes?", The Journal of Family Practice, vol.53, no.12, (2004), pp.974-980.

[5] J. W. Saultz and J. Lochner, "Interpersonal continuity of care and care outcomes: a critical review", Annual Family Medicine, vol.3, no.2, (2005), pp.159-166.

[6] J. H. Kim and H. J. Cho, "Effects of having regular source of care on preventive services and disease control", J Korean Academy of Family Medicine, vol.28, no.2, (2007), pp.78-85.

[7] "The Ministry of Health and Welfare", The Clinic Appointment System for systemic management of chronic disease management, (2011).

[8] "The Ministry of Health and Welfare", Plan for Implementation of Clinic Appointment System, (2011).

[9] J. R. Marc, W. Hsiao, P. Berman and M. R. Reich, "Getting Health Reform Right; A guide to improving performance and equity", Oxford university press, (2004), pp.97-134.

[10] M. R. Reich and D. Cooper, "PolicyMaker: Computer Aided Political analysis version 2.0", Brookline. M.A: PoliMap, (1996).

[11] A. Glassman, M. R. Reich and F. Rojas, "Political analysis of health reform in the Dominican Republic", Heath Policy and Planning, vol.14, no.2, (1995), pp.115-126.

[12] MediPana news. http://medipana.com/news, (2012).

[13] Pharmacy news. http://www.pharm21.com/news, (2011). 
[14] S. Kwon and M. R. Reich, "The changing process and politics of health policy in Korea", Journal of Health Politics, Policy and Law, vol.30, no.6, (2005), pp.1003-1025.

[15] B. D. Hwang, "The attitude of medical practitioners to the chronic disease care system", The Korean Journal of Health Service Management, vol.6, no.2, (2012), pp.193-200.

[16] I. H. Oh, "The healthcare system and chronic disease management system at outpatient clinic", The forum of Medical Policy, vol.10, no.4, (2012), pp.21-27.

\section{Author}

\section{Hyunsook Zin Lee}

Doctoral student, Department of Nursing,

Chung-Ang University

zinhslee@gmail.com 
International Journal of Bio-Science and Bio-Technology

Vol.7, No.4 (2015) 